\title{
Fourier Transform Multiple Quantum Nuclear Magnetic Resonance
}

\author{
By Gary Drobny, Alexander Pines, Steven Sinton, \\ DANIEL P. WeITEKAMP AND DAVID WeMMER \\ Department of Chemistry, \\ University of California, Berkeley, California, U.S.A.
}

Received 18th December, 1978

\begin{abstract}
The excitation and detection of multiple quantum transitions in systems of coupled spins offers, among other advantages, an increase in resolution over single quantum n.m.r. since the number of lines decreases as the order of the transition increases. This paper reviews the motivation for detecting multiple quantum transitions by a Fourier transform experiment and describes an experimental approach to high resolution multiple quantum spectra in dipolar systems along with results on some protonated liquid crystal systems. A simple operator formalism for the essential features of the time development is presented and some applications in progress are discussed.
\end{abstract}

The energy level diagram of a system of coupled spins $1 / 2$ in high field is shown schematically in fig. 1. The eigenstates are grouped according to Zeeman quantum number $m_{i}$ with smaller differences in energy within a Zeeman manifold due to the couplings between spins and the chemical shifts. For any eigenstate $|i\rangle$ of the spin Hamiltonian $H$ (in frequency units)

$$
\begin{aligned}
& H|i\rangle=\omega_{i}|i\rangle \\
& I_{z}|i\rangle=m_{i}|i\rangle .
\end{aligned}
$$

The single quantum selection rule ${ }^{1}$ of the low power c.w. experiment and the one dimensional Fourier transform experiment arises because $\left\langle i\left|I_{x}\right| j\right\rangle$ vanishes unless

$$
q_{i j}=m_{i}-m_{j}= \pm 1
$$

Simple combinatorial considerations show that the number of eigenstates decreases as $\left|m_{i}\right|$ increases and the number of transitions decreases as $\left|q_{i j}\right|$ increases. The highest order transition possible is the single transition with $|q|=2 I$ where $I$ is the total spin. For a system of $N$ spins $1 / 2$, transitions up to order $N$ are possible.

Detection of multiple quantum transitions in c.w. experiments is well known. ${ }^{2-4}$ Extension to high order transitions is not promising, since the transitions observed are a sensitive function of r.f. field strength. This leads to difficult spectral simulations and experimental problems of saturation and sample heating.

The alternative time domain experiment is the determination of multiple quantum transition frequencies by following the time development of multiple quantum coherences point by point.$^{5-7}$ This work treats a class of such multidimensional 


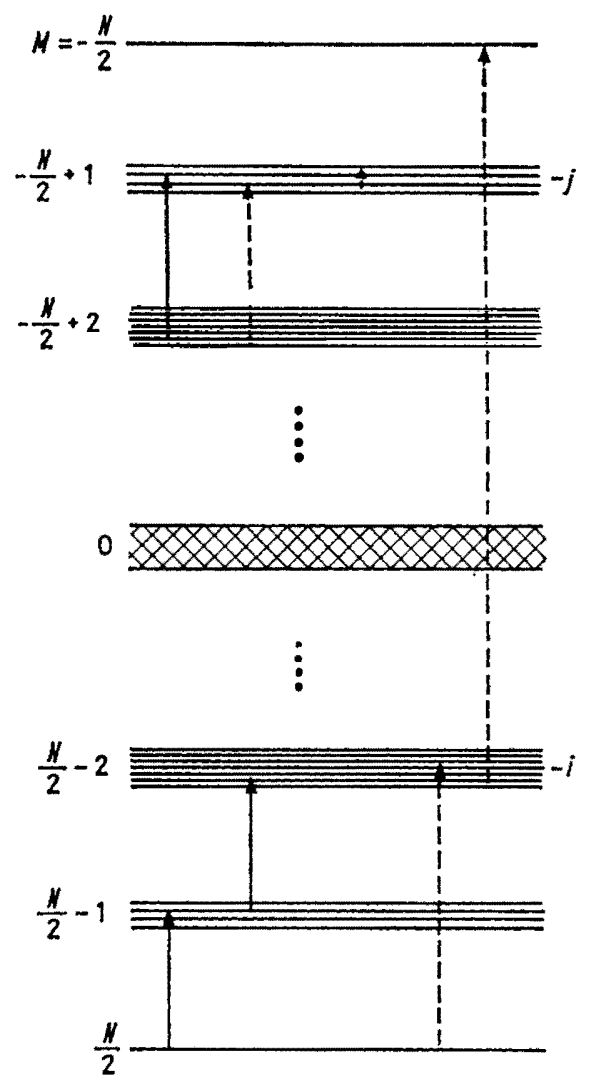

Fig. 1.--Schematic representation of the high field energy level diagram of coupled spins $1 / 2$. Broken arrows indicate the forbidden types of transition observed in Fourier transform multiple quantum experiments.

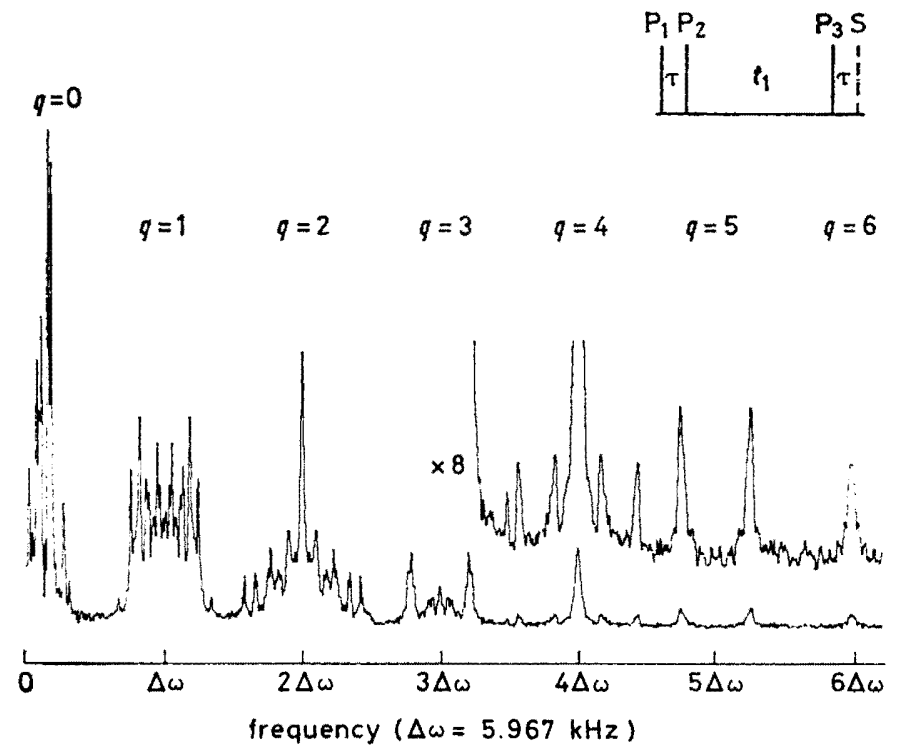

Fig. 2.-Multiple quantum spectrum of benzene $(15 \mathrm{~mol} \%)$ in p-ethoxybenzylidene-n-butylaniline (EBBA) at $20^{\circ} \mathrm{C}$. The three pulse sequence was $\mathrm{Pl}=\pi / 2_{x}, \mathrm{P} 2=\pi / 2_{\bar{x}}, \mathrm{P} 3=\pi / 2_{x}$. The magnitude spectra obtained for 11 values of $\tau$ spaced at $0.1 \mathrm{~ms}$ intervals from 9.6 to $10.7 \mathrm{~ms}$ were added. The value of $t_{1}$ ranged from 0 to $13.824 \mathrm{~ms}$ in $13.5 \mu$ sincrements for each $\tau$. A single sample point was taken at $t_{2}=\tau$ after $P_{3}$. One half of a symmetrical spectrum is shown. 
experiments in which the irradiation consists of pulses at the Larmor frequency. Time proportional incrementation of the r.f. phase (TPPI) allows separate determination of the spectra of all orders free from effects of magnet inhomogeneity.

\section{EXPERIMENTAL}

The spectrometer is of pulsed Fourier transform design with super-conducting magnet (Bruker) operating in persistent mode at a proton frequency of $185 \mathrm{MHz}$. Phase shifting was performed at $185 \mathrm{MHz}$ by a digitally controlled device (DAICO 100D0898) under control of the pulse programmer.

Samples were approximately $400 \mathrm{mg}$ sealed in $6 \mathrm{~mm}$ glass tubing after degassing by repeated freezing and evacuation. All observations are in the nematic phase. Synthesis of 4-cyano- $-4^{\prime}-\left[{ }^{2} \mathrm{H}\right]_{11}$ pentyl-biphenyl was by the procedure of Gray and Mosley. ${ }^{8}$

\section{RESULTS}

The spectrum of benzene dissolved in a liquid crystal served as a prototype in the development of the single quantum n.m.r. of complex spin systems in ordered phases. ${ }^{9}$ The multiple quantum spectrum of ordered benzene is shown in fig. 2 . The resolution is limited by magnetic homogeneity and the inhomogeneous linewidth is proportional to $|q|$.
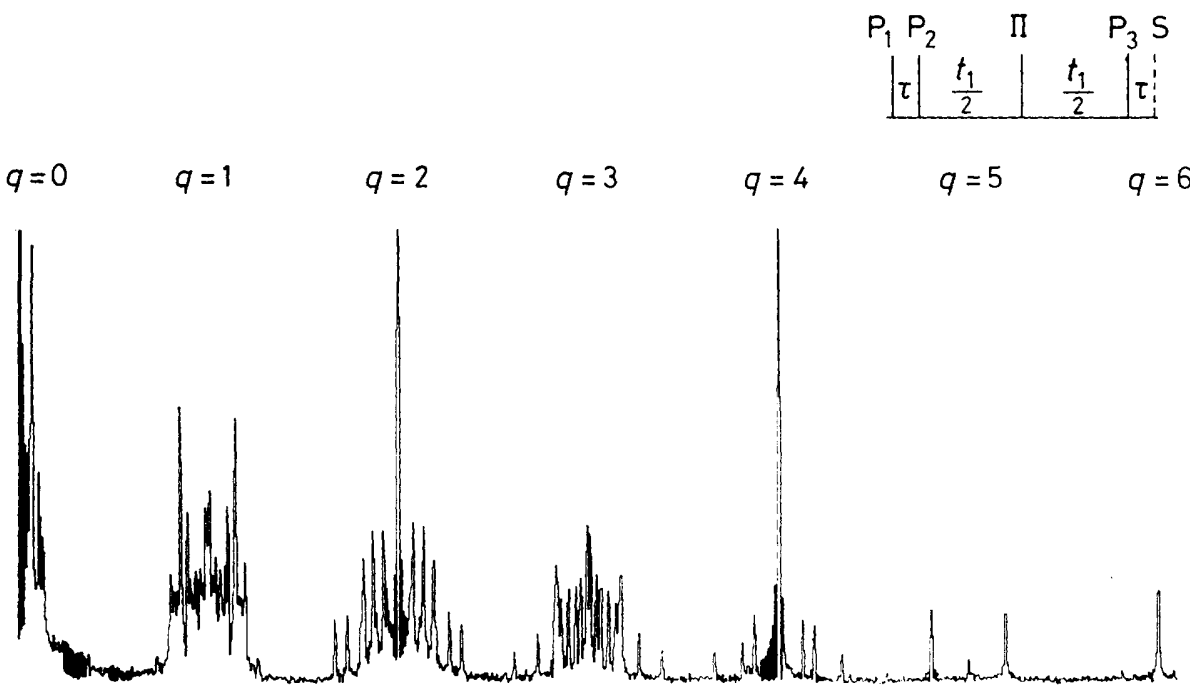

FIG. 3.--Multiple quantum spectrum of benzene at $22{ }^{\circ} \mathrm{C}$ and TPPI pulse sequence. The sample is the same as in fig. 2. The pulses are $\mathrm{P} 1=\pi / 2_{\varphi}, \mathrm{P} 2=\pi / 2 \bar{\varphi}$ and $\mathrm{P} 3=\pi / 2_{x}$, where $\varphi=\Delta \omega t_{1}$. The increment in $\varphi$ was 29.5 degrees and the increment in $t^{1}$ was $10 \mu \mathrm{s}$ for each of 1024 points. The magnitude spectra for eight values of $\tau$ between 9 and $12.5 \mu \mathrm{s}$ were added. The magnetization was sampled at $t=\tau$. 
The spectrum of fig. 3 demonstrates the use of the spin echo to remove inhomogeneous line broadening and the use of time proportional phase increments (TPPI) to restore the offset. Resolution is limited by truncation of the multiple quantum free induction decay and scale of reproduction. The actual linewidth is less than $2 \mathrm{~Hz}$ for all orders and suffices to resolve all allowed transitions of all orders.

An application of the TPPI method to the eight proton system of an alkyl-deuterated cyano biphenyl liquid crystal is shown in fig. 4. All eight orders are observed. Resolution is limited by truncation. Actual linewidths are $<100 \mathrm{~Hz}$ in a spectral width of $\approx 40 \mathrm{kHz}$ for each order.

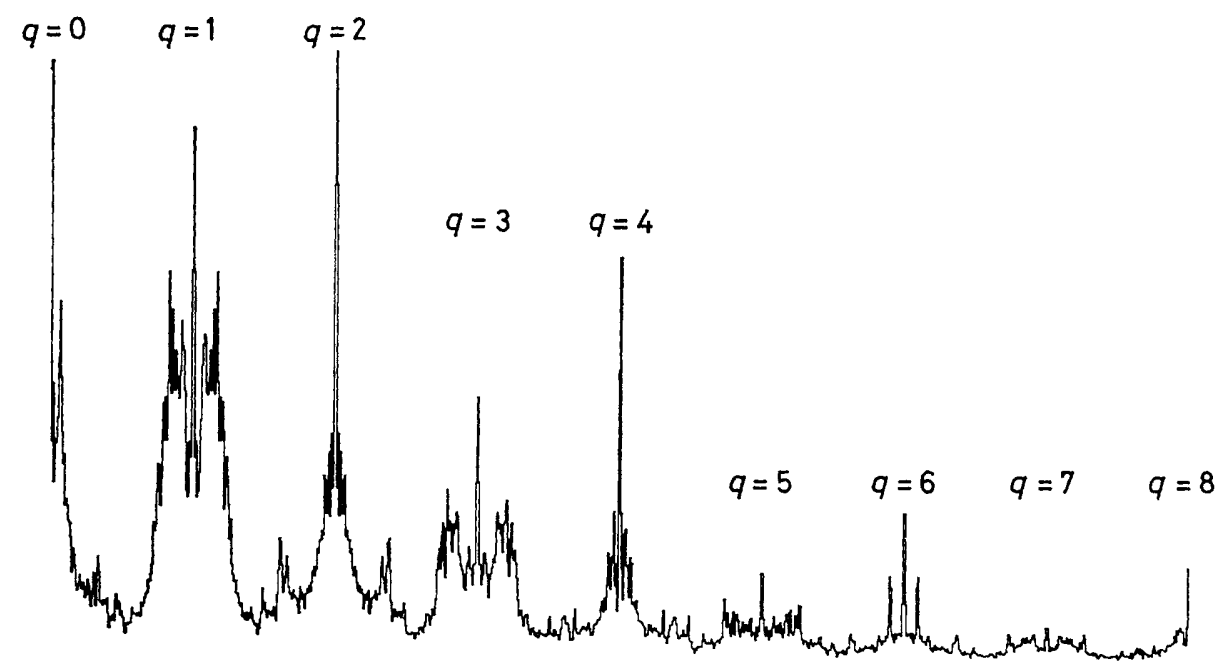

FIG. 4.-Proton multiple quantum spectrum of 4-cyano- $4^{\prime}-\left[{ }^{2} \mathrm{H}_{11}\right]$ pentyl-biphenyl at $23.3^{\circ} \mathrm{C}$ by TPPI. The increments are 22.5 degrees in $\varphi$ and $1.5 \mu \mathrm{s}$ in $t_{1}$ for each of 1024 points. The time $\tau$ took five values between 0.5 and $1.0 \mathrm{~ms}$ and the magnetization was sampled at 64 intervals of $5 \mu \mathrm{s}$ starting at $t_{2}=(\tau+0.1) \mathrm{ms}$.

\section{DISCUSSION}

\section{THREE PULSE EXPERIMENT}

The time development of the spin system during the pulse sequences of fig. 2 and 3 is conveniently discussed in terms of a spherical tensor operator expansion of the density matrix. For any time,

$$
\rho(t)=\sum_{k, \alpha, q} C_{q}^{k x}(t) T_{q}^{k \alpha}
$$

where $T_{q}^{k}$ is the $q$ th component of a spherical tensor operator of rank $k .^{10}$ The label $\alpha$ completes the specification of a complete basis of tensor operators.

The initial equilibrium density matrix is

$$
\rho(0)=C_{0}^{11}(0) I_{z}=C_{0}^{11}(0) T_{0}^{11}
$$

and immediately after a $\pi / 2_{y}$ pulse $\left(\omega_{1} \gg H, \omega_{1} t_{\mathrm{P} 1}=\pi / 2\right)$

$$
\rho\left(t_{\mathrm{P} 1}\right)=-C_{0}^{1 \mathrm{I}}(0) I_{x}=C_{1}^{1 \mathrm{I}}\left(t_{\mathrm{P} 1}\right) T_{1}^{1 \mathrm{I}}+C_{-1}^{1 \mathrm{I}}\left(t_{\mathrm{P} 1}\right) T_{-1}^{1 \mathrm{I}} \text {. }
$$


The Zeeman quantum number $q$ is conserved during evolution under a high field Hamiltonian. Thus neglecting relaxation

$$
\rho(t)=\sum_{k, \alpha, \pm 1} C_{q}^{k x}(t) T_{q}^{k_{\alpha}}
$$

at any time $t$ after the initial $\pi / 2$ pulse and no multiple quantum coherence has been created. After a period of time on the order of the inverse of the coupling frequencies, terms with $k \leqslant 2 I$ will be present. A second strong pulse may then rotate $T_{ \pm 1}^{k x}$ into $T_{q}^{k x}$ with $-k \leqslant q \leqslant k$. Thus at time $\tau$, after the second pulse, all orders with $-2 I \leqslant$ $q \leqslant 2 I$ have, in general, been created and evolve during the time $t_{1}$ at the eigenfrequencies $\omega_{i j}=\omega_{i}-\omega_{j}$.

If the only observable measured is the transverse magnetization corresponding in the rotating frame to the $|q|=1$ operators $I_{x}$ and $I_{y}$, it is not possible to follow the evolution of orders with $|q| \neq 1$ directly. Rather, a third pulse at time $t_{1}$ after the second pulse is needed to rotate the various tensor components back to $T_{ \pm 1}^{k x}$. These may then evolve into the signal observed at time $t_{2}$ after this detection pulse.

The signal may be written then as

$$
S\left(\tau, t_{1}, t_{2}\right)=C_{0}^{1 \mathbf{1}}(0) \operatorname{Tr}\left[I_{+} \rho\left(\tau+t_{1}+t_{2}\right)\right] .
$$

Viewed as a function of $t_{1}$ this is the multiple quantum free induction decay. It is collected pointwise by variation of $t_{1}$ on successive shots and is an example of a multidimensional n.m.r. experiment.

\section{SEPARATION OF ORDERS BY FREQUENCY OFFSET}

In order to obtain an increase in resolution over the single quantum experiments it is necessary to separate the spectra of different order. Consider the decomposition of the density matrix at time $\tau\left(t_{1}=0\right)$ into Zeeman components

$$
\rho(\tau)=\sum_{q=-2 I}^{2 I} \rho_{q}(\tau) .
$$

By construction, $\rho_{q}(\tau)=\sum_{k \alpha} C_{q}^{k \alpha}(\tau) T_{q}^{k \alpha}$ and it follows from the definition of the spherical tensor operators that

$$
\rho_{q}(\tau, \varphi)=\exp \left(\mathrm{i} \varphi I_{z}\right) \rho_{q}(\tau) \exp \left(-\mathrm{i} \varphi I_{z}\right)=\exp (\mathrm{i} q \varphi) \rho_{q}(\tau) .
$$

If we let $\varphi=\Delta \omega t_{1}$, then eqn (9) corresponds to a calculation of the effect of a frequency offset term $-\Delta \omega I_{z}$ in the rotating frame Hamiltonian. Thus a modulation by $\exp \left(\mathrm{i} q \Delta \omega t_{1}\right)$ is caused by the offset, shifting the spectrum to $q \Delta \omega$ as seen in fig. 2 .

The difficulty with this approach to separation of orders is that the effect of magnet inhomogeneity is proportional to $|q|$. The sample volume at position $\boldsymbol{r}$ with offset $\Delta \omega(\boldsymbol{r})$ contributes a term $\rho_{q}\left(\tau, t_{1}, \boldsymbol{r}\right)=\exp \left(\mathrm{i} q \Delta \omega(\boldsymbol{r}) t_{1}\right) \rho_{q}\left(\tau+t_{1}\right)$ and integration over $\boldsymbol{r}$ leads to damping of the coherence with a time inversely proportional to $|q|$.

\section{HIGH RESOLUTION SPECTRA BY TIME PROPORTIONAL PHASE INCREMENTS (TPPI)}

The Hahn spin echo technique may be used to remove the inhomogeneous broadening. A $\pi$ pulse at $t_{1} / 2$ changes $q$ to $-q$, allowing a refocusing of the coherence at $t_{1}$ for all orders. However, this $\pi$ pulse also removes the separation of the orders which was a result of the frequency offset. 
A solution to this dilemma is to view eqn (9) not as the effect of a frequency offset, but as a shift of the r.f. phase. In particular, consider the effect of preparing the system with pulses of phase $\varphi$ and $\bar{\varphi}=\varphi+\pi$ at time zero and $\tau$, respectively, with $I_{\varphi}=I_{x} \cos \varphi-I_{y} \sin \varphi$. Then

$$
\begin{aligned}
& \rho(\tau, \varphi)=\exp \left(\mathrm{i} \theta I_{\varphi}\right) \exp (-\mathrm{i} H \tau) \exp \left(-\mathrm{i} \theta^{\prime} I_{\varphi}\right) \times \\
& \rho(0) \exp \left(\mathrm{i} \theta^{\prime} I_{\varphi}\right) \exp (\mathrm{i} H \tau) \exp \left(-\mathrm{i} \theta I_{\varphi}\right) \\
& =\exp \left(\mathrm{i} \varphi I_{z}\right) \exp \left(\mathrm{i} \theta I_{x}\right) \exp (-\mathrm{i} H \tau) \exp \left(-\mathrm{i} \theta^{\prime} I_{x}\right) \times \\
& \rho(0) \exp \left(\mathrm{i} \theta^{\prime} I_{x}\right) \exp (\mathrm{i} H \tau) \exp \left(-\mathrm{i} \theta I_{x}\right) \exp \left(-\mathrm{i} \varphi I_{z}\right) \\
& =\exp \left(\mathrm{i} \varphi I_{z}\right) \rho(\tau) \exp \left(-\mathrm{i} \varphi I_{z}\right) \\
& =\sum_{q} \exp (\mathrm{i} \varphi \varphi) \rho_{q}(\tau) .
\end{aligned}
$$

The result is that again

$$
\rho_{q}(\tau, \varphi)=\exp (\mathrm{i} q \varphi) \rho_{q}(\tau)
$$

but this modulation is now an artifact of the r.f. phase of the first two pulses and does not depend on the evolution of the system during $t_{1}$. However, since each point $t_{1}$ of the signal is collected separately, we may set $\varphi=\Delta \omega_{t i}$ for some parameter $\Delta \omega$, thereby recovering an apparent offset.

The actual evolution during $t_{1}$ may include an echo pulse to remove the effect of field inhomogeneity, as in fig. 3 , or a train of $\pi$ pulses to remove the effect of small chemical shifts and heteronuclear couplings from the dipolar spectra.

This TPPI technique may be compared to the method of phase Fourier transformation (PFT) discussed elsewhere. ${ }^{11,12}$ The PFT method generates a signal array $S\left(\tau, t_{1}, t_{2}, \varphi\right)$ by repeating the experiment for each $t_{1}$ and $\varphi$, where again $\varphi$ describes the phase of the preparation pulses as in eqn (10). Fourier transformation with respect to phase with $q$ as the conjugate variable separates the orders. A second Fourier transformation with respect to $t_{1}$ gives the spectra. In the TPPI experiment the phase and time dimensions are collapsed into one by the relation $\varphi=\Delta \omega t_{1}$. A single time Fourier transformation gives the spectrum of each order with apparent offset $q \Delta \omega$ as in fig. 3 and fig. 4.

\section{MULTIPLE QUANTUM SPECTROSCOPY OF LIQUID CRYSTALS}

Although the spectroscopy discussed is of general applicability, it is worthwhile to note the particular suitability of the m.f.t.n.m.r. method to the study of liquid crystals. The diffusion present in such systems sharply reduces the intermolecular dipolar couplings. This allows one to obtain resolved spectra reflecting only the intramolecular couplings without dilution of the spin system. Combinatorial arguments suggest that it suffices in general to analyse only the $|q|=N-2$ and $N-1$ spectra to determine all couplings in a system of $N$ spins $1 / 2$. Since these transitions involve only the relatively small $|m|=\frac{N}{2} \frac{N}{2}-1$ and $\frac{N}{2}-2$ Zeeman manifolds of the basis of kets, the diagonalizations are simpler than those needed for single quantum spectroscopy. Should the study of mixtures be of interest, partial deuteration of the background species will reduce its contribution to the high order spectra more rapidly than to the single quantum spectrum and thus the requirement for isotopically pure synthesis is reduced.

Applications in progress include the configurational analysis of both ring and chain regions of liquid crystals, the study of relaxation effects in multiple quantum spectra 
and improvement of signal to noise by removal of the dipolar Hamiltonian during detection and sampling of magnetization at many times $t_{2}$.

We would like to acknowledge the help of Mr. Sidney Wolfe in synthesis of the liquid crystals and of Terry Judson in preparation of the manuscript. Support for this work was by the Division of Materials Sciences, Office of Basic Energy Sciences, U.S. Department of Energy.

D.P.W. held a Predoctoral National Science Foundation Fellowship.

${ }^{1}$ I. J. Lowe and R. E. Norberg, Phys. Rev., 1957, 107, 46.

${ }^{2}$ W. A. Anderson, Phys. Rev., 1956, 104, 850.

${ }^{3}$ J. I. Kaplan and S. Meiboom, Phys. Rev., 1957, 106, 499.

${ }^{4}$ K. M. Worvill, J. Mag. Res., 1975, 18, 217.

${ }^{5}$ (a) H. Hatanaka and T. Hashi, J. Phys. Soc. Japan, 1975, 39, 1139.

(b) H. Hatanaka, T. Terao, and T. Hashi, J. Phys. Soc. Japan, 1975, 39, 835.

${ }^{6}$ W. P. Aue, E. Bartholdi and R. R. Ernst; J. Chem. Phys., 1976, 64, 2229.

${ }^{7}$ (a) S. Vega, T. W. Shattuck and A. Pines, Phys. Rev. Letters, 1976, 37, 43.

(b) S. Vega and A. Pines, J. Chem. Phys., 1977, 66, 5624.

${ }^{8}$ G. W. Gray and A. Mosley, Mol. Cryst. Liq. Cryst., 1976, 35, 71.

9 A. Saupe, Z. Naturforsch, 1965, 20a, 572.

${ }^{10}$ A. R. Edmonds, Angular Momentum in Quantum Mechanics (Princeton University Press, Princeton, N.J.; 2nd Edn. 1960), Chap. V, p. 68.

11 A. Wokaun and R. F. Ernst, Chem. Phys. Letters, 1977, 52, 407.

12 A. Pines, D. Wemmer, J. Tang and S. Sinton, Bull. Amer. Phys. Soc., 1978, 23, 21. 